\title{
GENERANDO IDENTIDADES COLECTIVAS EN ESPACIOS URBANOS RECONFIGURADOS
}

\section{COLLECTIVE IDENTITIES IN RECONFIGURED URBAN SPACES}

\author{
Diego Nápoles Franco \\ Universidad de Guadalajara \\ dienafra@hotmail.com
}

Resumen: En este trabajo se trata de materializar la identidad colectiva, retomando los conceptos de memoria y cultura como proveedoras de significados y destacando el papel de los colectivos en relación a las "ciudades medias" transformadas a metrópolis, partiendo del análisis teórico-conceptual de otros autores, con el objetivo de construir aproximaciones metodológicas para su aplicación a casos de estudio concretos; como las sociedades que habitan espacios urbanos de mediano tamaño, expuestos a transformaciones socioespaciales continuas, por factores de crecimiento económico-globales.

Palabras clave: Identidad colectiva, memoria colectiva, cultura, ciudad media, espacio urbano reconfigurado.
Abstract: : In this communication, we try to gain a clear understanding of collective identity, returning to the concepts of memory and culture as providers of meaning and highlighting the role of groups in relation to "medium cities" transformed into metropolises. Our approach has been to start from theoretical-conceptual analyses of other authors with the objective of building methodological approaches for its application to specific case studies such as societies that inhabit urban spaces of medium size and are exposed to continuous socio-spatial transformations due to factors of global economic growth.

Key words: Collective identity, colletive memory, culture, medium city, and urban space reconfigured. 


\section{Introducción}

En la actualidad, las grandes ciudades se consolidan a través de espacios globalizados con vinculaciones que superan barreras y obstáculos de crecimiento en la era post-moderna (Giménez 1996; Castells, 1999; Bauman, 2006; Harvey, 2012). Las zonas urbanas de menor tamaño, como el caso de las llamadas "ciudades medias", no están exentas a esta dinámica, sin embargo, son distintas las características socioeconómicas de estas urbanizaciones, debido a diferentes posicionamientos y capacidades financieras, políticas y geográficas, principalmente en el mercado mundial. Los habitantes de estos entornos presentan variaciones en sus identidades individuales y colectivas no sólo por sus particulares formas culturales, sino por los constantes cambios estructurales en sus actividades relacionadas a una nueva época ${ }^{1}$. En principio, se aborda esta situación de cambio de las identidades colectivas y su definición, para continuar con algunas explicaciones teóricas referentes a la memoria y la cultura. Posterior a ello, el estudio se enfoca en la identificación y discusión de variables e indicadores pertinentes para su aplicación en sociedades que habitan las "ciudades medias metropolitanas" en crecimiento, finalizando con reflexiones acerca del abordaje de las identidades colectivas en espacios urbanos reconfigurados.

\section{La situación de las identidades colectivas urbanas en la actualidad. Modificación o mantenimiento en el espacio urbano reconfigurado}

En otras investigaciones documentadas, se advierte sobre los cambios sociales y urbanos de quienes habitan las ciudades, debido a procesos económico-globales principalmente (Hurtado, 2010; Montaner y Muxí, 2011; Harvey, 2012). De esta manera, las relaciones sociales se ven afectadas por procesos de cambio urbano y funciones capitalistas fuertemente posicionadas en países desarrollados y subdesarrollados (Harvey, 2012) 2 .

En el mundo, existen ciudades que muestran un crecimiento económico importante, gracias a la inserción del capital y la vinculación con mercados internacionales (Garrocho y Sobrino, 1995; Castells, 2006; Harvey, 2012; Casado y Sánchez, 2013). Lo anterior, no sólo se ve reflejado en la transformación del espacio físico, sino también en sus sociedades. La ciudad media borra cada vez más su pasado de influencia rural para convertirse en una nueva metrópoli regional, donde su ensanchamiento urbano trae implícito otros aspectos de cambio, como nuevas conductas sociales

1 De acuerdo con otras tesis, los resultados generados por distintos procesos económicos como el fordiso- postfordismo, capitalismo-postcapitalismo y en general de la globalización y liberación de los mercados generan cambios sociales y nuevas dinámicas socio-económicas en un nuevo espacio universal, retomando fuerza en una era llamada post-moderna en el siglo XXI, donde se continúa con la constante evolución de los procesos económicos (Castells, 1999; Castells, 2006; Harvey, 2012).

2 Partimos del análisis de las ciudades, no solo en contextos económico-globales relacionados con el neo-liberalismo y el post-capitalismo, sino en la continua transformación urbana de ciudades mexicanas caracterizadas por su diversidad cultural y condición de pobreza (Castells, 1999; Lezama, 1993). 
y formas de construir el hábitat (Harvey, 2012) ${ }^{3}$. Estas sociedades se caracterizan por tener identidades colectivas relacionadas con la función de su territorio, como espacio regulador de migración hacia las grandes áreas metropolitanas y de posicionamiento como polos de crecimiento y desarrollo regional (Pasciaroni, 2012; Venegas, 2003). Estas características particulares radican en las influencias que las grandes ciudades ejercen sobre las ciudades medias y los entornos rurales cercanos (Sassen, 2006). En la mayoría de las ciudades latinoamericanas, se pueden encontrar identidades significativas no sólo por los contenidos culturales, sino por las actividades que se realizan en ellas, e incluso por la condición de pobreza que les caracteriza (Lezama, 1993) Para el estudio de las identidades colectivas de las ciudades medias, en procesos de transformaciones urbanas, es también importante tomar en cuenta las conductas relacionadas con factores de tipo económico:

Una de las características de las sociedades contemporáneas es que, a pesar del aumento general de los ingresos per capita, también aumentan los riesgos y las fragilidades. Todo ello a base de construcción de las ciudades a borrar la memoria urbana con el objetivo de debilitar las redes sociales y comunitarias, que podrian oponerse a determinados proyectos urbanos y por tanto económicos. Entramos aquí en otro de los entresijos de los procesos contemporáneos de urbanización, la disolución de la memoria plural y compleja, un mecanismo político que pretende imponer nuevas identidades colectivas, concepciones simples y manipuladas de lo social (Montaner y Muxí, 2011: 159).

Según algunos casos de la actualidad, las sociedades son dirigidas por agentes políticos cuyos objetivos se centran en la captación de recursos económicos para la transformación de las ciudades, a través de obras que reconfiguran el espacio (Soja, 2000). En este proceso de cambio urbano, la demanda de trabajo y vivienda son factores determinantes. En consecuencia, las identidades colectivas también se ven afectadas por las nuevas dinámicas de las relaciones sociales y patrones de consumo en entornos globalizados (Hurtado, 2010) ${ }^{4}$. Las nuevas identidades colectivas se acompañan de intenciones de control y debilitamiento de las redes sociales, agudizando la exclusión social y donde el principal interés se centra en la inserción de capitales privados:

La globalización ha agudizado la descomposición de los hilos o lazos que dan sentido a la identidad: tradiciones, usos, costumbres, ritos, leyendas, mitos, creencias, religiones, y una pluralidad de memorias que reivindican orígenes y valores propios, contrapuestos a los que enarbolan otros grupos o memorias colectivas. Proyectos que por los resultados hasta ahora conocidos resultan contrarios no solo a la identidad sino también a la cultura y a la unidad, lo cual ha perturbado tanto al desarrollo nacional como al regional y local (Hurtado, 2010: 117).

En esta ocasión, se analiza el comportamiento de las identidades colectivas de algunas ciudades medias mexicanas en procesos de transformación urbana constante (SEDESOL, CONAPO e INEGI 2010). Se considera como elementos relevantes para las identidades colectivas de estos entornos urbanos: su ambiente económico en

3 La función que deriva de la jerarquización de los espacios urbanos se relaciona con la preponderancia de ciertas ciudades con respecto a otros sitios de menor conglomeración e incluso rurales (Soja, 2000), esto genera una mezcla de dinámicas y relaciones en el espacio social en distintas formas y entre distintos niveles espaciales como nuevas formas de apropiación del territorio, usos de suelo y construcción de vivienda entre otros (Sassen, 2006).

4 Siguiendo esta hipótesis, podemos encontrar diversas comunicaciones como las de Pérez Astete (2011) y de Lapresta y Huguet ( 2006). 
crecimiento, pobreza y diversidad cultural predominante. Estas características abren un debate irresuelto sobre el mantenimiento de las identidades colectivas. Por un lado, se mantienen debido a la alta concentración de materiales culturales en sus sociedades y, por otro, la vulnerabilidad de modificarse por posibles intervenciones e influencias de otras áreas con mayor desarrollo ${ }^{5}$. Por consiguiente, se cree necesario discutir algunas de las aportaciones teórico-conceptuales para futuras investigaciones y selección de casos de estudio.

\section{Identidad colectiva. La definición del nosotros en relación a los otros y el sentido de pertenencia}

Para comprender la identidad como proceso de reconocimiento fundamental de los individuos, es necesario entender que este término se apoya con la definición del nosotros en relación a los otros (Giménez, 1997; Giménez, 2008; Bourdieu, 2006). En este proceso de identificación, el individuo no sólo analiza los elementos de juicio y valor que considera propios o compartidos, sino las diferencias con respecto a otros actores sociales o colectivos. Se retoma la aportación que Giménez hace al respecto:

La identidad está relacionada con la idea que tenemos acerca de quiénes somos y quiénes son los otros, es decir, con la representación que tenemos de nosotros mismos en relación con los demás. Implica, por tanto, hacer comparaciones entre las gentes para encontrar semejanzas y diferencias entre ella (Giménez, 2008: 6).

El análisis que las personas realizan en base a las diferencias y semejanzas, se traduce en la necesidad de reconocerse dentro de la estructura social, con el objetivo de identificar también el papel de cada actor en determinados contextos. De ahí la importancia de este trabajo, centrado en la definición de las identidades colectivas urbanas en contextos globales, en base a los conceptos de memoria y cultura. En este trabajo, la cultura resulta ser el componente común, donde se contienen los significados para el proceso de distingo de las personas. Sin embargo, se pretende situar este análisis a través de tres dimensiones espaciales urbanas propuestas por Soja como intraurbana, interurbana y de jerarquía, mismas que se nombran como DAU's ${ }^{6}$. En relación a ello, los elementos o significados de la cultura, se presentan como materiales culturales, los cuales se ubican en la sociedad, es ahí donde las personas recurren para abonar a la producción de la identidad (Giménez, 2008). Cabe mencionar, que la gente relaciona estos datos de acuerdo con su estructura social y los colectivos a los que pertenece. Además de la reconfiguración de las identidades colectivas en base a ciertos significados compartidos, también es necesario el reconocimiento por parte de otros grupos, aunque no es fundamental para su consolidación y existencia (Giménez, 1997). Por su parte, Bourdieu considera que las

5 Los materiales culturales abonan al fortalecimiento de las identidades colectivas, sin embargo, existe la posibilidad del debilitamiento de las mismas a través de nuevas intervenciones que construyen otras formas de entender el mundo y por consecuencia de una actuación diferente a la anterior (Giménez, 1996; Giménez, 2008). Al respecto, no pretendemos realizar calificativos, pero sí tratar de adentrarme en el estado de las identidades colectivas urbanas de la actualidad y su futura dirección.

6 De aquí en adelante, se hará referencia a las dimensiones de análisis del espacio urbano como DAU's. Partiendo de la definición de los individuos que habitan la ciudad en cuestión, a través de las dimensiones: 1) intraurbana, de la zona centro en comparación a otras zonas del interior de la ciudad; 2) interurbana, de la ciudad central con respecto a otras ciudades de menor o igual tamaño, y 3) jerárquica, en relación a otros asentamientos de distintos tamaños en la región como áreas rurales y metropolitanas (Soja, 2000). 
identidades colectivas se caracterizan por compartir diferentes símbolos de carácter subjetivo, como emblemas e insignias, entre otros, los cuales son percibidos y apreciados de manera individual pero compartiendo ciertas generalidades, destacándose las más importantes para ser portadas como grupo. En este acontecimiento también vale la participación de otros sujetos que realizan valorizaciones al respecto, pero todo a través de representaciones (Bourdieu, 2006).

Por su parte, Giménez reconoce las identidades segregadas como aquéllas que se afirman sin importar el reconocimiento que otras realicen con respecto a su existencia. También utiliza el término de "heterodirigidas", las cuales se caracterizan por la débil capacidad de auto- reconocimiento, aunque sí existe la identificación por los demás y menciona a las identidades etiquetadas, cuando el actor se auto-identifica, aunque su diversidad haya sido fijada por otros (Giménez, 1997). También es importante tomar en cuenta el significado del factor "tiempo", para cualquier proceso de identidad, en los acontecimientos y materiales culturales. Aunque es también necesario mencionar que la identificación del "nosotros", tiene la condición de reconocer a los demás (Bourdieu, 2006; Giménez, 1997; Giménez, 2008). En lo que respecta sobre la identidad del sujeto individual, nos referimos a un autoanálisis como actor social; en consecuencia, una valoración de los otros y del entorno. Es entonces necesaria no sólo una autorreflexión, sino hacer el contraste con otros sujetos sociales para dar paso al reconocimiento público en un ambiente social de relaciones. Es decir, la definición de grupo en relación a otros, llamada por otros teóricos "identidad espejo", que no es más que el resultado de cómo nos ven los demás (Giménez, 2008). Esto nos dice que en la acción de identificar, va implícito el proceso de identificar a otros sujetos presentes en el entramado social. La identidad contiene fundamentos individuales pero también colectivos, esto a través de los materiales compartidos socialmente (Giménez, 1997; Giménez, 2008). En ese sentido, se incluye la pertenencia social como otra característica sobresaliente al momento de darse el proceso de identidad de un individuo o colectivo.

El hombre pertenece socialmente a diversos grupos sociales según sus intereses, con esto se pretende poner en contexto, la situación relacional que el individuo tiene en base a círculos sociales, donde pone en práctica la identidad propia y de los otros con los que interactúa y se relaciona de manera continua. La pertenencia social imprime la característica de apego en la identidad, esto se puede observar en el ejercicio cotidiano de los individuos, al mostrar su relación con ciertos objetos, personas o diversas prácticas cotidianas y conductas de consumo lo que conlleva en la creación de una etiqueta particular. Para la creación de esta etiqueta propia, el individuo también recurre a estilos de vida en relación con ciertos intereses, que a su vez se enlazan con otros individuos (Giménez, 2008). La pertenencia social se relaciona con las representaciones sociales compartidas entre los miembros de un grupo, las mismas que se traducen a diferentes creencias, opiniones, puntos de interés, conocimientos compartidos, entre otros, con los cuales se construye la realidad común colectiva, aunque no todos los miembros del grupo comparten en el mismo grado las representaciones sociales (Giménez, 1997).

En relación, el sentimiento de pertenencia es primordial para las identidades colectivas, porque a través de él es como se mueven y se vinculan sus miembros. Aunque la "acción colectiva", no siempre es característica de las identidades colectivas. Por su parte, Bourdieu se refiere a la importancia del reconocimiento de los colectivos con el objetivo de institucionalizarse en una sociedad que se afirma a través de representaciones y voluntades; propone que, para existir socialmente, habrá que ser percibido como distinto (Bourdieu, 2006). Al respecto, la postura de Yi-Fu Tuán 
(2007) en relación al sentido de pertenencia, se liga con procesos afectivos, cognitivos y de interacción, donde las personas se reconocen en el entorno, dándose así una autoatribución del mismo como parte de su identidad.

\section{La memoria colectiva como recurso generador de compo- nentes inclusivos para la formación de la identidad}

La memoria colectiva no es menos importante en la formación de las identidades, pues resulta ser un componente más que abona a su construcción, a través de los sucesos registrados en la historia de las sociedades ${ }^{7}$. Los momentos considerados como significativos en determinado espacio y tiempo colaboran en la configuración de las identidades colectivas como una especie de proveedores que sin su existencia la memoria estaría nula o borrada. La memoria puede ser un recurso individual o colectivo según quien la utilice, retome o porte. De ahí la importancia de la espacialidad, donde Bourdieu, por ejemplo, habla de la región (como elemento característico de apego). Por otra parte, Giménez se refiere en general a la territorialidad como un elemento espacial, donde las identidades colectivas se enmarcan (Giménez, 2008). Existe literatura en relación al apego amoroso entre individuos y territorio, donde se elabora un lenguaje para entender la identidad nacional en base a la lógica que se establece entre madre e hijo, sinónimo de tierra-habitante (Vivar, 2002).

Al respecto, los recuerdos en las ciudades se pueden observar en espacios creados simbólicamente para representar determinadas culturas. La cultura en lo general proporciona elementos clave para que los individuos que conforman un colectivo se desarrollen. Las identidades colectivas requieren de la creación de memorias colectivas a diferencia de las memorias biográficas que caracterizan a las identidades individuales. Lo anterior con la finalidad de reforzar su historia y permanencia como colectivo. En consecuencia, la identidad individual tiene la función de autoidentificarse y al mismo tiempo de lograr el reconocimiento de los otros, pero las identidades colectivas no resuelven este aspecto. En otras palabras, no necesariamente requieren del reconocimiento de otras identidades como enseguida se expresa:

Resulta imposible hablar de identidad colectiva sin referirse a su dimensión relacional. Vista de este modo, la identidad colectiva comporta una tensión irresuelta e irresoluble entre la definición que un movimiento ofrece de sí mismo y el reconocimiento otorgado al mismo por el resto de la sociedad (Giménez, 2008: 13-14).

Enseguida se tratará de poner a la mesa otros elementos que sin lugar a dudas servirán de plataforma para la construcción de una estructura conceptual de las identidades colectivas urbanas. Como vemos, el entramado social requiere de una exploración compleja para acercarnos a la realidad, mayormente cuando ocurren grandes cambios en contextos económico-globales como en el que se sitúa este trabajo. Además, indagando en la memoria colectiva, se obtendrá un acercamiento hacia otros aspectos representativos del lugar e incluso de personajes históricos y de importante significado para la configuración de la identidad.

7 Diversas investigaciones demuestran la importancia de los datos históricos o contenidos de la memoria colectiva, donde se afirma su colaboración en la construcción de las identidades colectivas (nacionales, regionales, institucionales entre otras) como las realizadas por Lapresta (2006), Del Olmo (2003), Vivar (2002) o Infante y Hernández (2001). 


\section{La cultura como proveedora de significados para la con- solidación de la identidad}

Se considera que la cultura es una fuente sólida que alimenta la identidad continuamente, no sólo porque en ella descansan los contenidos que caracterizan el comportamiento de la sociedad, sino las formas a las cuales obedece y que incluso pueden referirse a otros periodos previos (Díaz Guerra, 2010). Así, la identidad tiene su fuente en la cultura, entendiéndose como organización social.

...la organización social de significados, interiorizados de modo relativamente estable por los sujetos en forma de esquemas o de representaciones compartidas, y objetivados en formas simbólicas, todo ello en contextos históricamente específicos y socialmente estructurados (Giménez, 2008: 3).

Se coincide con el autor en proponer a la cultura como una dimensión que comprende ámbitos sociales, económicos, políticos, etc. de toda la esfera social. No obstante, deberá existir un trabajo donde se identifiquen los contenidos para la elaboración de la cultura, como proveedora de significados en la construcción de la identidad, tomando en cuenta otros aspectos analíticos, de acuerdo a las distintas formas de percibir, concebir y vivir el espacio, de acuerdo con la memoria colectiva y la cultura ${ }^{8}$. Como una aproximación a la definición de la variedad de significados de la cultura, se sugiere nombrarla como una estructura social objetiva donde los contenidos muestran una amplia gama de representaciones y símbolos que se presentan a través de la historia y donde los agentes sociales toman relevancia. Estos significados se muestran a través de formas simples y complejas en el entramado social a lo largo de distintos periodos, es decir, se habla de formas culturales y comportamientos durante cortos o largos espacios temporales u otras construcciones simbólicas, como obras de arte, ritos y danzas que representan el contenido social, abonando a la elaboración de la identidad (Giménez, 2008).

Entiéndase lo anterior, como una clara expresión de la realidad, donde no se puede pensar respecto a la existencia de un sujeto sin cultura. Es en este aspecto donde el ser humano toma el papel central de la estructura social objetiva, retomando ciertos significados de su propia cotidianeidad, costumbres y comportamientos de la cultura para posteriormente identificarse con la misma. Sin embargo, se plantea necesaria una temporalidad específica para que la cultura sea significativa, en otras palabras, se habla de la estabilidad de los elementos o significados en los colectivos o individuos (Giménez, 2008). Esta propuesta, está basada en la relación entre individuos y colectivos-tiempo y espacio ${ }^{9}$, donde el actor social o los colectivos participan en el desarrollo de los elementos culturales que a priori o posteriori se vuelven significados. Lo anterior, no equivale a la resistencia de los significados culturales a la inmovilidad, por el contrario, la cultura no es del todo estática:

8 De acuerdo al método aportado por Eduard Soja (2000), basado en tres modos de investigar la ciudad y resultados de las interconexiones entre los espacios percibidos, concebidos y vividos, a los cuales se les denomina como modos generales de investigación MGI. Este modelo consiste en analizar el espacio desde la percepción, concepción y la manera en que se vive por parte de los actores sociales.

9 La cultura se contiene, desarrolla y afirma por actores sociales de manera individual o colectiva, dentro de una estructura social a través de una temporalidad determinada, haciendo uso de los recursos culturales por medio de interrelaciones temporales y de la memoria (Giménez, 1996; Giménez, 2008). 
La cultura nunca debe entenderse como un repertorio homogéneo, estático e inmodificable de significados. Por el contrario, puede tener a la vez "zonas de estabilidad y persistencia" y "zonas de movilidad" y cambio (Giménez, 2008: 5).

Se pretende exponer que la cultura no sólo toma el papel como proveedora de significados para la identidad colectiva, sino que también funge como una estructura de valores construidos, de acuerdo con diferentes aspectos de carácter espacial y temporal, donde intervienen procesos cualitativos en determinados contextos, como el que hoy nos ocupa. Por otra parte, es oportuno tomar en cuenta la posibilidad de la conservación de las identidades colectivas a pesar de la existencia de variaciones en la cultura ${ }^{10}$.

En el siguiente apartado, se presenta el concepto de "cultura" como denominador común entre los sujetos individuales y aquéllos que conforman los colectivos, tratando de incluir los aspectos valorativos propios de cada entidad. A su vez, se integra la "memoria" para concretar una valoración de los componentes esenciales, para el análisis de las identidades colectivas y dar paso a una co-estructura metodológica.

\section{Presentación conceptual de las identidades}

En primer orden, se muestra la estructura conceptual de la memoria como factor determinante para el abordaje de las identidades colectivas, indicando la variabilidad de los hechos retomados de la misma. En relación a la antropología, se establecen dos tipos de memorias: la fuerte o "masiva" también como coherente, compacta y profunda en donde se generan las identidades de mayor fuerza (Giménez, 2008), por ejemplo, la memoria de las instituciones religiosas. En lo que respecta a las memorias débiles, entiéndase aquellos hechos superficiales que difícilmente se comparten por un grupo de personas. Este tipo de memorias, se caracterizan por ubicarse en las grandes ciudades ${ }^{11}$.

\begin{tabular}{|c|c|c|c|c|}
\hline Concepto & \multicolumn{3}{|c|}{ Identidad } \\
\hline Categoría & \multicolumn{3}{|c|}{ Memoria } \\
\hline Variables & \multicolumn{2}{|c|}{ Memorias fuertes } & \multicolumn{2}{c|}{ Memorias débiles } \\
\hline Indicadores & $\begin{array}{c}\text { Reproducción } \\
\text { recuerdos }\end{array}$ & $\begin{array}{c}\text { Representaciones } \\
\text { producidas }\end{array}$ & Relatos breves & $\begin{array}{c}\text { Representaciones } \\
\text { producidas cortas }\end{array}$ \\
\hline Valor observable & \multicolumn{2}{|c|}{ Hechos trascendentales } & \multicolumn{3}{|c|}{ Hechos de menor importancia } \\
\hline Categoría & \multicolumn{3}{|c|}{ Cultura } & \multicolumn{2}{|c|}{ Visión del mundo } \\
\hline Variables & \multicolumn{2}{|c|}{ Comunicación } & \multicolumn{2}{c|}{ Conocimientos } \\
\hline
\end{tabular}

10 Siguiendo la hipótesis de Giménez (1996:26), "salvo catástrofe o genocidio, las culturas y las identidades tradicionales de origen étnico o mestizo-campesino no se disuelven ni cambian dramáticamente al contacto con la modernidad (por lo menos en el curso de una generación), sino solo se transforman adaptativamente enriqueciéndose, redefiniéndose articulándose con ella".

11 Recordemos que este trabajo es propuesto para el abordaje de ciudades medias en procesos de transformación (metropolitano) además de las particulares características antes mencionadas. De tal manera que se consideran estos núcleos de población con capacidades culturales suficientes que servirán de recurso para su análisis. 


\begin{tabular}{|c|c|c|c|}
\hline \multirow{2}{*}{ Concepto } & \multicolumn{2}{|c|}{ Identidad } \\
\hline \multirow{2}{*}{ Indicadores } & Símbolos y signos & Creencias & Religión \\
& Hábitat & Contemplación & Filosofía \\
\hline \multirow{2}{*}{ Valor observable } & Alimentación & Sentido común & Ideología \\
\hline
\end{tabular}

Cuadro 1. Tratamiento conceptual "memoria y cultura".

Fuente: Elaboración propia, basado en Giménez (2008).

En este apartado, se toman como preponderantes a aquellos hechos de mayor peso para los colectivos. Es decir, la sistematización de diferentes situaciones de acuerdo a su importancia y la relación con la consolidación de la identidad colectiva. A partir de la identificación y valorización de los materiales considerados como trascendentales, se dimensionara su relevancia, sometiendo la información a través de distintos filtros analíticos que previamente se definieron como sistemas MGI y DAU basándose en Soja (2000).

\begin{tabular}{|c|c|c|c|c|c|c|c|c|c|}
\hline Categoría & \multicolumn{9}{|c|}{ Memoria } \\
\hline Sub-categoría & \multicolumn{9}{|c|}{ Hechos trascendentales } \\
\hline Variables & \multicolumn{3}{|c|}{ ¿Cómo se perciben? } & \multicolumn{3}{|c|}{ ¿Cómo se conciben? } & \multicolumn{3}{|c|}{ ¿Cómo se viven? } \\
\hline Indicador* & 1 & 2 & 3 & 1 & 2 & 3 & 1 & 2 & 3 \\
\hline
\end{tabular}

* Indicadores urbanos DAU: 1 intraurbanos, 2 interurbanos y 3 jerárquico.

Cuadro 2. Procesamiento de los hechos trascendentales.

Fuente: Elaboración propia, basado en Giménez (2008) y Soja (2000).

En base a las capas arriba señaladas, se busca obtener la valorización de los colectivos a través de tres cuestionamientos, en relación al espacio urbano como a continuación se detalla:

1. Intraurbanos. Percepciones, concepciones y vivencias de hechos trascendentales en la ciudad.

2. Interurbanos. Percepciones, concepciones y vivencias de hechos trascendentales de otras ciudades contiguas.

3. Jerárquico. Percepciones, concepciones y vivencias de hechos trascendentales de entornos rurales y áreas urbanas de mayor tamaño en la región.

De acuerdo con esta propuesta, es necesario posicionar a los MGI como variables y a las DAU como indicadores para facilitar su uso en relación a las categorías y subcategorías identificadas. De la misma manera, se traslada la estructura conceptual propuesta para el tratamiento de los materiales culturales de referencia como enseguida se muestra en el cuadro 3. 


\begin{tabular}{|c|c|c|c|c|c|c|c|c|c|}
\hline Categoría & \multicolumn{9}{|c|}{ Cultura } \\
\hline Sub-categoría & \multicolumn{9}{|c|}{ Referencias culturales vigentes } \\
\hline Variable & \multicolumn{3}{|c|}{$\begin{array}{l}\text { ¿Cómo se perciben } \\
\text { culturalmente? }\end{array}$} & \multicolumn{3}{|c|}{$\begin{array}{c}\text { ¿Cómo se conciben } \\
\text { culturalmente? }\end{array}$} & \multicolumn{3}{|c|}{$\begin{array}{l}\text { ¿Cómo se viven } \\
\text { culturalmente? }\end{array}$} \\
\hline Indicador* & 1 & 2 & 3 & 1 & 2 & 3 & 1 & 2 & 3 \\
\hline
\end{tabular}

* Indicadores urbanos DAU: 1 intraurbanos, 2 interurbanos y 3 jerárquico.

Cuadro 3. Procesamiento de las referencias culturales vigentes.

Fuente: Elaboración propia, basado en Giménez (2008) y Soja (2000).

Según los resultados de los procesos de tratamiento de la memoria y cultura, se estará en condiciones de construir aproximaciones conceptuales del "nosotros" y el "ellos". Para este proceso, es necesario entender el "nosotros" como colectivo(s) de la ciudad y el área metropolitana y el "ellos" como colectivos de zonas rurales y otras ciudades 12.

\begin{tabular}{|c|c|c|c|c|}
\hline Categoría & \multicolumn{4}{|c|}{ Definición de "nosotros" } \\
\hline Variables & \multicolumn{2}{|c|}{ Ciudad } & \multicolumn{2}{|c|}{ Área metropolitana } \\
\hline Indicadores & $\begin{array}{c}\text { Memoria } \\
\text { intraurbana }\end{array}$ & $\begin{array}{c}\text { Cultura } \\
\text { intraurbana }\end{array}$ & $\begin{array}{c}\text { Memoria } \\
\text { intraurbana e } \\
\text { interurbana }\end{array}$ & $\begin{array}{c}\text { Cultura } \\
\text { intraurbana e } \\
\text { interurbana }\end{array}$ \\
\hline Observable & \multicolumn{2}{|c|}{ Definición de "nosotros ciudad" } & \multicolumn{2}{|c|}{$\begin{array}{l}\text { Definición de "nosotros ciudad } \\
\text { metropolitana }\end{array}$} \\
\hline Categoría & \multicolumn{4}{|c|}{ Definición de "ellos" } \\
\hline Variables & \multicolumn{2}{|c|}{ Rurales } & \multicolumn{2}{|c|}{ Ciudad (otras) } \\
\hline Indicadores & $\begin{array}{l}\text { Memoria } \\
\text { Jerárquico }\end{array}$ & $\begin{array}{c}\text { Cultura } \\
\text { Jerárquico }\end{array}$ & $\begin{array}{c}\text { Memoria } \\
\text { interurbana }\end{array}$ & $\begin{array}{c}\text { Cultura } \\
\text { interurbana }\end{array}$ \\
\hline Observable & \multicolumn{2}{|c|}{ Definición de "ellos rurales" } & \multicolumn{2}{|c|}{ Definición de "ellos ciudad" } \\
\hline
\end{tabular}

Cuadro 4. Tratamiento de las identidades colectivas en áreas urbanas.

Fuente: Elaboración propia, basado en Giménez (2008) y Soja (2000).

Atendiendo los resultados generados a través de los procesos ilustrados en los cuadros 2 y 3 e integrando las referencias relevantes compartidas de la memoria y la cultura, se intentarán obtener las siguientes definiciones:

- "Nosotros ciudad", en base a la dimensión intraurbana.

- "Nosotros área metropolitana", resultado de la alianza entre dimensión intraurbana e interurbana.

- "Ellos rurales", apoyados de la dimensión nivel jerárquico.

- "Ellos ciudad(es) contigua(s)", utilizando la dimensión interurbana.

En referencia a la construcción de ambas definiciones, se destacan las aportaciones de los individuos, basadas en una valorización como identidades colectivas, ubi-

12 En este caso, se hace referencia a las ciudades cercanas, situadas en la misma región. 
cadas en la ciudad transformada a zona metropolitana, en relación a aquéllos que habitan áreas rurales y urbanas contiguas de distinto tamaño.

\section{Consideraciones finales}

El tratamiento conceptual expuesto en este documento, es sólo una propuesta general para el abordaje de las identidades colectivas, situadas en ciudades medias reconfiguradas como áreas metropolitanas. Por lo anterior, se recomienda realizar las adecuaciones necesarias según sean los objetivos específicos de otras investigaciones urbanas. Con este trabajo, se intenta realizar una introducción al entramado social, para conocer cómo se construyen las identidades colectivas que habitan en los espacios urbanos, en constante crecimiento y exposición al desarrollo económico-global. Sin embargo, es necesario recurrir a otros apoyos metodológicos que se centran específicamente en los informantes, quienes representan a los colectivos.

Por otra parte, es oportuno identificar los materiales culturales compartidos y los hechos significativos guardados en la memoria, para obtener una radiografía de las identidades colectivas en la ciudad. Esto sólo se genera mediante el análisis de los colectivos y de su entorno, como componentes particulares según el caso que se seleccione. En relación, consideramos importante la participación de los individuos en la ciudad, no sólo por el sentido de pertenencia que se genera, sino por las relaciones que a lo largo de la historia se han ejercido, fundado y mantenido, a través de actividades que forman a los individuos como a través de los materiales culturales y que pueden abonar a la construcción del nosotros y el ellos.

No sólo las identidades colectivas se rigen por la cultura y la memoria, sino por el grado de participación que tienen dentro de la estructura social y el apego al territorio. Sin embargo, será para otras producciones teórico-analíticas que se retomará este aspecto. Por tanto, también es necesario, indicar que la cultura tiene ramificaciones con las cuales se construyen las formas de identidad. Dicho de otra manera, la cultura no participa, pero sí es proveedora de significados, los cuales son determinantes en la elaboración de las identidades con respecto a un lugar específico.

Para futuros estudios, se sugiere investigar el grado de relación existente entre los individuos que comparten un espacio urbano como la ciudad, la colonia y el barrio, entre otros. De esta manera, se podrá tener un acercamiento a las similitudes y diferencias de las identidades colectivas en distintos núcleos espaciales urbanos.

De acuerdo con Giménez Gilberto, compartimos la idea de la relevancia de las formas de vida por parte de los grupos, para la configuración de las primeras nociones de identidad colectiva. Razón por la cual se concluye que el peso de las identidades colectivas lo proporcionan los materiales culturales y la memoria colectiva, propios de cada grupo situado en un determinado entorno también contribuyente. En el contexto mexicano, las ciudades medias sufren de cambios constantes en su estructura socio-económica, debido a influencias por parte de grandes metrópolis, lo cual genera una interconexión entre diferentes escalas de núcleos urbanos y rurales, propiciando su continua expansión y generando nuevas categorías de ciudad, además de nuevas formas de construir el hábitat y de otros aspectos que se vuelven complejos, como el uso de suelo, la movilidad y las relaciones sociales.

El estudio de la fórmula socio-urbana compuesta por las identidades colectivas y el espacio urbano reconfigurado permite un acercamiento hacia la manera en que los colectivos se identifican con el nuevo tamaño de la ciudad. Por ello, se toma la apli- 
cación de la propuesta que consiste en identificar el caso de estudio y su contexto social, económico, político y cultural, para después seleccionar las dimensiones urbanas del mismo y de su entorno y, posteriormente, dar paso al tratamiento de la memoria y la cultura, estableciendo una línea de tiempo para analizar el estado de las identidades colectivas y su vinculación con el espacio físico. Por lo tanto, la presente es sólo una herramienta de apoyo socio-urbano, para el estudio de las identidades de grupos que habitan las ciudades medias contemporáneas en continua expansión.

\section{Bibliografía}

Bauman, Z. (2006). Modernidad Líquida. México: F.C.E.

Bourdieu, P. (2006). La identidad y la representación: elementos para una reflexión crítica sobre la idea de región. Ecuador debate, 67, 165-184.

Casado Izquierdo, J. M. y Sánchez Salazar, M. T. (2013). Coatzacoalcos: Reestructuración urbana e inversión privada en una ciudad media mexicana. Revista EURE, v. 39, $\mathrm{n}^{\circ} 117$, mayo, 91-116.

Castells M. (1999). La Cuestión urbana. Madrid: Siglo XXI.

Castells, M. (2006). La Sociedad Red: Una Visión Global. Madrid: Alianza Editorial.

Del Olmo, N. (2003). Construcción de identidades colectivas entre inmigrantes: ¿Interés, reconocimiento y/o refugio?". Reis, 104/03, 29-56.

Díaz, S. y Guerra, R. (2010). Construcción de la identidad de grupo local - el espacio y el patrimonio material e inmaterial como referentes identitarios en Trujillo y Huertas de Ánimas. Gazeta de Antropología, 26 (2).

Garrocho, C. y Sobrino, J. (coord.) (1995). Sistemas metropolitanos. Nuevos enfoques y prospectiva. Secretaria de Desarrollo Social. El Colegio Mexiquense, A.C.

Giménez, G. (1996). Territorio y cultura. Estudios sobre las culturas contemporáneas, v. II, $n^{\circ} 4,9-30$.

Giménez, G. (1997). Materiales para una teoría de las identidades sociales. Frontera Norte, v. 9, $n^{\circ} 18$, julio-diciembre.

Giménez, G. (2008). Cultura, identidad y memoria. Materiales para una sociología de los procesos culturales en las franjas fronterizas. UNAM.

Harvey, D. (2012). El enigma del capital y la crisis del capitalismo. Madrid: Akal.

Hurtado Santiago, M. (2010). Los cristales de la identidad y la cultura: La multiculturalidad como forma de conservación. En (In)justicia Social, identidad e (in)equidad. Retos de la modernidad. Universidad Autónoma Metropolitana.

Infante, M. E. y Hernández, C. (2001). Preservar la identidad cultural: Una necesidad en la actualidad. ASRI Arte y sociedad, $\mathrm{n}^{\circ} 0$.

Lapresta y Huguet (2006). Identidad colectiva y lengua en contextos pluriculturales y plurilingües. El caso del valle de Arán. Revista Internacional de Sociología (RIS), v. LXIV, $n^{\circ} 45,83-115$.

Lezama, J. (1993). Teoría social, espacio y ciudad. México: El colegio de México. 
Montaner, J. M. y Muxi, Z. (2011). Arquitectura y Política. Ensayos para mundos alternativos. Barcelona: Gustavo Gili.

Pasciaroni, C. (2012). Ciudades medias: Aproximación metodológica, funcionalidades y estructura productiva. Revista Ciencias Económicas, v. 30, nº 1.

Pérez Astete, F. (2011). Globalización y nuevas identidades culturales: identidad de género en construcción. Revista Sociedad \& Equidad, 2.

Sassen, S. (2006). Territory. Authority. Rights. From Medieval to Global Assemblages. Princeton University Press.

Sedesol, Conapo e Inegi (2010). Delimitación de las zonas metropolitanas de México.

Soja, E. W. (2000). Postmetrópolis. Estudios críticos sobre las ciudades y las regiones. España: Creative Commons.

Tuán, Y. F. (2007). Topofilia. Un estudio de las percepciones, actitudes y valores sobre el entorno". Barcelona: Editorial Melusina.

Venegas, C. (2003). Efectos del crecimiento de la industria mueblera en la estructura urbana de Ocotlán Jalisco: 1989-1999. Carta Económica Regional, 86.

Vivar, F. (2002). Primeras señas de identidad colectiva: las alabanzas de España medievales. Castilla, 27, 141-158.

Zapiain Aizpuru, M. T. (2011). Reflexiones identitarias en el territorio contemporáneo. La construcción colectiva de lugar. Caso de estudio de la Vega de Granada. Cuadernos Geográficos, 48, 79-108. 
